\title{
Review Article \\ Murine Models of Nonalcoholic Fatty Liver Disease and Steatohepatitis
}

\author{
Masashi Ninomiya, Yasuteru Kondo, and Tooru Shimosegawa \\ Division of Gastroenterology, School of Medicine, Tohoku University, Sendai 9808574, Japan \\ Correspondence should be addressed to Yasuteru Kondo; yasuteru@ebony.plala.or.jp
}

Received 30 October 2012; Accepted 22 November 2012

Academic Editors: A. M. Concejero, C. Domenicotti, and B. Radosevic-Stasic

Copyright (C) 2013 Masashi Ninomiya et al. This is an open access article distributed under the Creative Commons Attribution License, which permits unrestricted use, distribution, and reproduction in any medium, provided the original work is properly cited.

In 1980, Ludwig et al. first reported patients of steatohepatitis who lacked a history of excessive alcohol consumption but showed liver histology resembling alcoholic hepatitis and progression to cirrhosis of the liver accompanied by inflammation and fibrosis. The development of nonalcoholic steatohepatitis (NASH) is associated with obesity, diabetes mellitus, insulin resistance, and hyperlipidemia. However, the pathogenesis of NASH remains incomplete. A "multiple-hit" hypothesis for the pathogenesis of NASH based on an animal model has been proposed and remains a foundation for research in this field. We review the important dietary and genetic animal models and discuss the pathogenesis of NASH.

\section{Introduction}

Although, until recently, fatty liver had been thought to take a benign clinical course, it is now known that nonalcoholic fatty liver disease (NAFLD) or NASH may progress to cirrhosis of the liver accompanied by inflammation and fibrosis [1]. It appears that the prevalence of simple steatosis is from $20 \%$ to $30 \%$ and in NASH from $2 \%$ to $3 \%$ in adults, and that NAFLD rarely leads to cirrhosis (3\%) compared to NASH (up to 30\%) [2]. Obesity, diabetes mellitus, insulin resistance, and hyperlipidemia are conditions frequently associated with NASH $[3,4]$.

The liver histology of NASH was confirmed by Brunt and colleagues and the NASH Clinical Research Network $[5,6]$. The diagnostic standards include the extent of steatosis, hepatocellular inflammation, and fibrosis. In steatosis, microvesicular and macrovesicular lipid droplets can be revealed in the whole cytoplasm. In contrast to simple steatosis, in steatohepatitis (NASH) there is inflammation with the presence of steatosis, hepatocellular ballooning, and both lobular and portal inflammation with fibrosis. Moreover, pericellular fibrosis with collagen secreted from hepatic stellate cells is a typical formation that can spread to the portal area and lead to portal-portal and portal-central bridging fibrosis and cirrhosis [7].
The development and progression of NAFLD to NASH would occur via a "two-hit" process involving the interaction of genetic and environment factors $[8,9]$. In brief, the progression from normal, healthy liver to steatohepatitis is a gradual process involving, first, the advance of obesity and insulin resistance and, next, inflammation [10]. However, this "two-hit" hypothesis remains controversial. In general, about $30 \%$ of patients in the spectrum of NAFLD develop $\mathrm{NASH}$, while patients with only steatosis tend to remain stable over time [11].

To understand the cause of NASH, several factors should be considered, and animal models may be useful as well as taking into account the previous reports. In this paper, we describe animal models and discuss the etiology and pathogenesis of NAFLD and NASH (Table 1).

\section{The Pathogenesis of NASH}

The pathogenesis of NASH remains incompletely understood. The use of animal models has been indispensable to clarifying the mechanisms by which NASH develops. Indeed, Day and James have proposed a "two-hit" hypothesis for the pathogenesis of NASH based on an animal model that remains a foundation for research in this field [8]. However, 
TABLE 1: Dietary models of nonalcoholic fatty liver disease.

\begin{tabular}{|c|c|c|c|c|c|}
\hline Model & Obesity & Insulin resistance & Steatohepatitis & Hepatic fibrosis & Elevated AST and ALT \\
\hline MCD diet & Weight loss & Hepatic insulin resistance & Yes & Yes & Yes \\
\hline $\begin{array}{l}\text { Choline-deficient, L-amino } \\
\text { acid defined (CDAA) diet }\end{array}$ & Weight loss & No & Yes & Yes & Yes \\
\hline Atherogenic diet & $-^{*}$ & Hepatic insulin resistance & Yes & Yes & - \\
\hline High-fat diet & Weight gain & Yes & Yes & Slight & - \\
\hline Fructose diet & - & Yes & No & No & Yes \\
\hline Adiponectin null & Weight gain & Yes & Yes & Yes & Yes \\
\hline Sfrp5 knockout & Weight gain & Yes & Yes & - & - \\
\hline Bid null & Weight gain & Yes & Yes & - & - \\
\hline Fas adipocyte-specific knockout & Weight gain & Yes & No & - & - \\
\hline IL-6 and TNF null & - & - & No & - & - \\
\hline LIKK & No change & Yes & No & No & No \\
\hline $\mathrm{NEMO}^{\mathrm{L}-\mathrm{KO}}$ & Weight gain & Yes & Yes & - & - \\
\hline $\mathrm{JNK}^{-/-}$ & Weight gain & Yes & No (only steatosis) & No & - \\
\hline
\end{tabular}

*Indicates no data.

this "two hit" hypothesis is known to involve interactions between insulin resistance, adipokines, adipose tissue inflammation, and other pathogenetic factors. Particularly, it has been proposed that hepatic steatosis may be the source of many distinct injurious factors rather than simply a "first hit" [12]. For this reason, the classical "two-hit" hypothesis of progression to NASH is now being modified by a "multiple parallel hits" hypothesis [13]. In the "multiple parallel hits" model, the first step is insulin resistance. Hyperinsulinemia, caused by insulin resistance, leads to steatosis by the accumulation of fat in the liver due to the increased inflow of free fatty acids (FFA) or de novo lipogenesis. Furthermore, when adaptive mechanisms for stress tolerance are overwhelmed, lipotoxicity and chronic inflammation activate major hepatic injury via oxidative and inflammatory stress and lipid peroxidation [9]. Consequently, all these processes contribute to the development of steatohepatitis, fibrosis, and cancer.

Several mechanisms related to the initial accumulation of fat in the liver have been reported. The impairment of lipid metabolism develops by metabolic syndrome with obesity and excessive oral intake of fats and carbohydrates. The overabundance of FFA also results in fat accumulation. In the field of clinical medicine, protein-calorie malnutrition, jejunoileal bypass, and parenteral nutrition sometimes result in abnormal liver function with triglyceride accumulation by affecting lipoprotein synthesis and causing the inhibition of lipid export from the liver $[14,15]$. Excessive triglyceride and the uptake of FFA by the liver induce insulin resistance. Insulin resistance is found in most patients with NAFLD and has been observed in patients who are not obese and have normal glucose tolerance. Moreover, adipokines derived from adipose tissue, such as adiponectin, leptin, and resistin, can be factors in the development of NASH mainly via their effects on insulin resistance $[16,17]$. Such multiple factors encompass the interaction of injurious processes including mitochondrial dysfunction and oxidative stress, cytokine-mediated liver injury, altered lipid partitioning and hepatotoxicity mediated by FFA, abnormal intrahepatic cholesterol loading, hyperinsulinemia, hyperleptinemia, and hypoadiponectinemia and apoptosis, which can lead to necroinflammation and, ultimately, fibrosis in the liver (Figure 1) [18, 19]. All of these steps interact in complicated ways to enhance the progression of hepatic lesions through the NAFLD spectrum.

Recently, NASH and simple fatty liver have been suggested to be two independent conditions, and a prospective study showed that the progression of simple fatty liver to NASH is uncommon [20]. Therefore, although simple fatty liver and NASH are considered to be two histological subtypes of NAFLD, these entities may be different not only from a histological, but also from a pathological view point.

\section{Dietary Animal Models}

3.1. Methionine and Choline Deficiency (MCD) Diet. The obese $(o b)$ gene was reported to regulate the energy balance in mouse by Zhang et al. Leptin gene deficiency $(o b / o b)$ leads to obesity and type II diabetes, which resembles morbid obesity in humans, including NAFLD [21]. A similar clinical condition has been described in $d b / d b$ mouse, which lacks the leptin receptor. Hepatic steatosis and the early stage of inflammatory changes in the liver have been shown in both of these strains of mice, but the MCD diet is required to increase the serum alanine aminotransferase (ALT) levels and develop prominent steatosis in zone 3 (pericentral) and the subsequent necroinflammation which are similar to what occurs in human NASH $[22,23]$. The MCD diet promotes intrahepatic lipid accumulation by increasing fatty acid uptake and decreasing very low density lipoprotein (VLDL) production. The expression of triglyceride synthesisrelated genes is downregulated [24]. Activated macrophages infiltrate into the liver of MCD-fed mice. The activation of nuclear factor $-\kappa \mathrm{B}(\mathrm{NF}-\kappa \mathrm{B})$ has been demonstrated together with concomitant increases in the induction of 


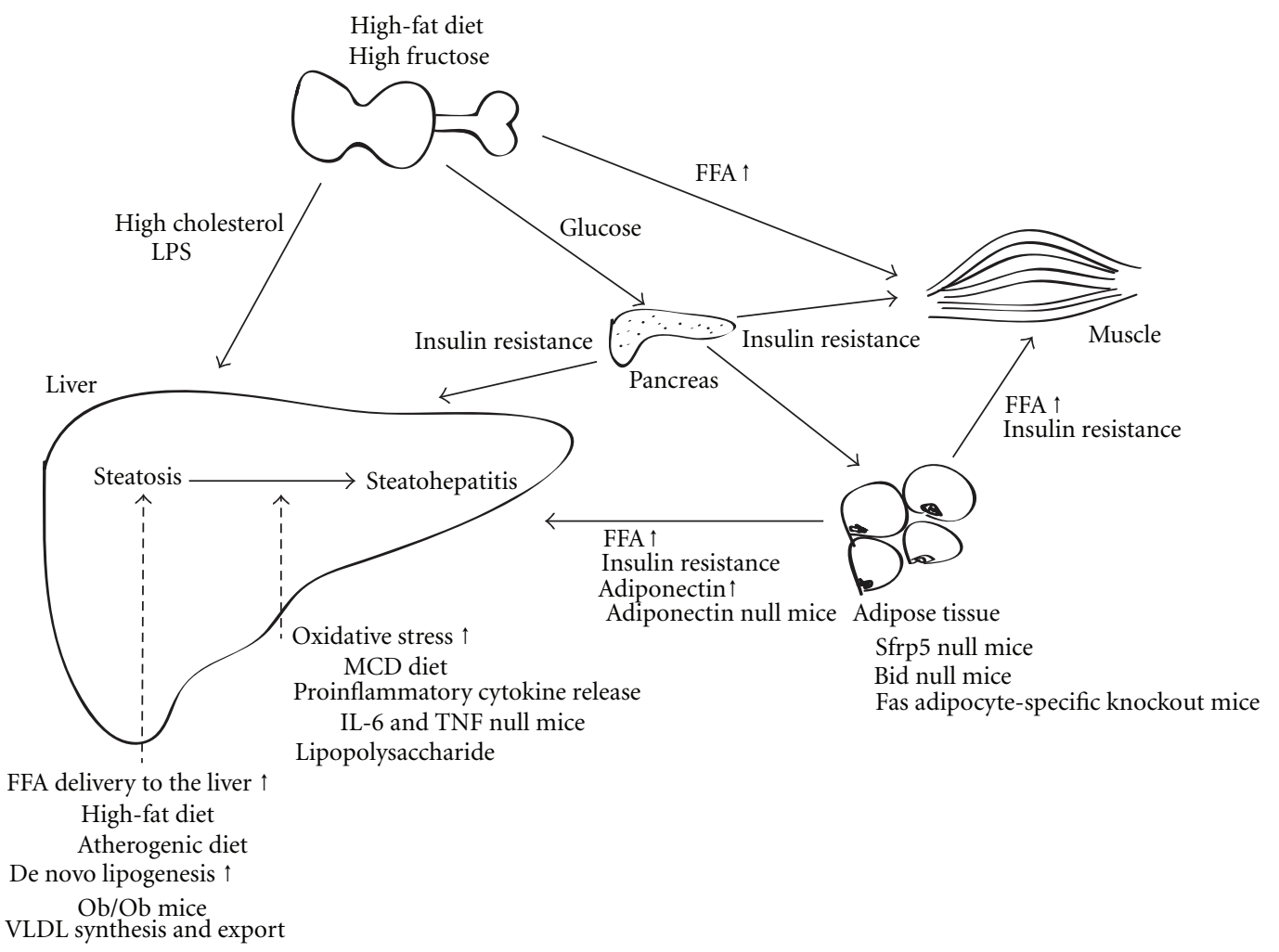

FIGURE 1: The "multiple parallel hits" hypothesis for the pathogenesis on NASH and the pathophysiological characteristics of animal models. The intake of a high-fat and high-fructose diet and overnutrition lead to metabolic syndrome and obesity. The progression to steatohepatitis in liver is in a stepwise manner involving first the development of fatty changes and, later, hepatic inflammation. Some of the animal models and pathogenic processes are also summarized.

proinflammatory intercellular adhesion molecule-1 (ICAM1), cyclooxygenase-2 (COX-2), interleukin 6 (IL-6), transforming growth factor- $\beta$ (TGF- $\beta$ ), and tumor necrosis factor (TNF) mRNA [25-27]. Although the MCD diet is considered to produce a NASH model, actually the plasma triglyceride and cholesterol levels are decreased, and the metabolic profile is the opposite of that seen in NASH patients. That is to say, serum adiponectin is increased and the insulin, and glucose levels are decreased $[28,29]$.

\subsection{Choline-Deficient, L-Amino Acid Defined (CDAA) Diet.} The CDAA diet has been used to produce rodent models of steatosis and liver cell death. The serum aspartate and alanine aminotransferase levels are elevated, but CDAA diet rats do not grow fat or show increased insulin resistance [30].

3.3. Atherogenic Diet. Matsuzawa et al. fed mice an atherogenic (Ath) diet containg $1.25 \%$ cholesterol and $0.5 \%$ cholate and reported the progression of steatosis, inflammation, and fibrosis in the liver [31]. Concerning the etiology of the progression to steatohepatitis, it was confirmed that free cholesterol loading caused TNF- and Fas-induction [32]. The mice became obese and showed increased levels of triglycerides and muscle insulin resistance [33]. The hepatocellular ballooning, a characteristic histopathological finding in human $\mathrm{NASH}$, was observed in the mouse livers. Furthermore, gene expression analysis revealed that the Ath diet upregulated the hepatic expression levels of genes for fatty acid synthesis, oxidative stress, inflammation, and fibrogenesis, and downregulated those of antioxidant enzymes [31].

3.4. High-Fat Diet. Animal models fed a high-fat diet, in which the majority of caloric intake is from fat, show metabolic syndrome with fatty liver formation and insulin resistance. A rat model fed a high-fat diet (71\% of energy from fat, $11 \%$ from carbohydrates, and $18 \%$ from protein) develops mild steatosis. Several effects such as insulin resistance, elevation of serum insulin, hepatic lipid accumulation, oxidative stress, and abnormal mitochondria have been reported [34]. The rats became obese and showed abnormal aminotransferase activity similar to that in human NASH. Moreover, immunoblot analysis showed that the expression of CYP2E1 was increased, whereas PPAR- $\alpha$ was reduced [35].

3.5. Fructose. The increased consumption of high fructose, primarily in the form of soft drinks, is reported to be a risk factor for the development of NAFLD in human. Ingestion of fructose promotes de novo lipogenesis, ATP depletion, and insulin resistance $[36,37]$. Mice fed with $30 \%$ fructose water showed high levels of hepatic triglycerides and a marked increase in steatosis and weight. Further investigation has shown that dietary fructose intake promotes gut-derived 


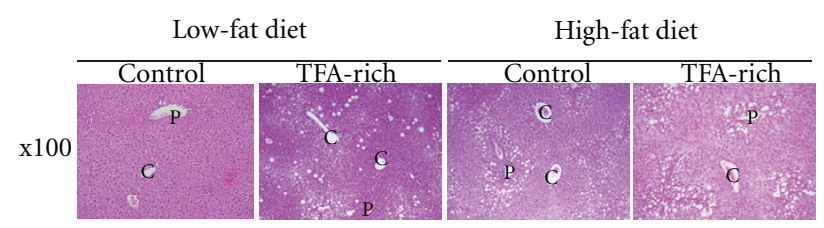

FIGURE 2: Representative liver histology stained with hematoxylin and eosin stains. The $\mathrm{C}$ indicates the central vein, and the $\mathrm{P}$ indicates the portal vein.

endotoxemia in the portal blood and then the activation of Kupffer cells and hepatic inflammation. Of note, Spruss et al. reported that the mice model of fructose-induced NAFLD is associated with intestinal bacterial overgrowth and increased intestinal permeability, leading to an endotoxindependent activation of hepatic Kupffer cells. So, Toll-like receptor 4 (TLR4) knockout mice show decreased levels of steatohepatitis, suggesting that fructose overconsumption can cause hepatic damage [38].

3.6. Trans Fatty Acid (TFA) Diet Mice. Trans fatty acid (TFA), primarily found in partially hydrogenated vegetable oils, is used by the food industry to make products more stable and robust. Its intake is known as a high risk factor for cardiovascular disease, insulin resistance, and obesity [39, 40]. We prepared a low-fat diet (LF $11.8 \%$ fat/total nutrition) and high-fat diet (HF 63.6\% fat/total nutrition) made of either natural canola oil as the control oil (LF-C and HF-C) or partially hydrogenated canola oil as the TFA-rich oil $(28.5 \%$ TFA/total fat, LF-T, and HF-T), respectively. Four groups of mice were fed these diets for 24 weeks.

Concerning the physiological characteristics, the body weight was increased in HF-fed mice, compared to LFfed mice and HF-T-fed mice weighed 1.3-fold more than HF-C-fed mice. Some serum markers were elevated in the HF-T group compared to the HF-C group, particularly alanineaminotransferase, triglyceride, total cholesterol, (V)LDL-cholesterol, FFA, and leptin were significantly increased. As for the control oil-fed mice, total cholesterol, HDL cholesterol, (V)LDL cholesterol, and adiponectin were lower, whereas plasma leptin was higher in HF-C-fed than in LF-C-fed mice. In contrast, no significant difference was found between LF-C-fed and LF-T-fed mice.

Few lipid droplets appeared in the LF-fed mice liver, while there were abundant, large lipid droplets in HF-C-fed mouse livers. However, the livers of HF-T-fed mice were characterized by foamy, prominent microvesicular steatosis. Marked, small lipid droplets that surrounded the hepatocytes, inflammation and ballooning degeneration and neutrophil infiltration were expanded in the liver tissue (Figure 2). Moreover, an early fibrosis marker, collagen type1, the $\alpha 1$ mRNA level and lipid accumulation in the livers of HF-Tfed mice were increased. An in vitro study demonstrated that TFA intake increased TNF- $\alpha$ production and intensified the phagocytotic ability of Kupffer cells [41].

In summary, TFA-rich oil intake, in addition to a high fat diet, induces severe steatosis and liver injury.

\section{Genetic Models}

4.1. Adiponectin Null Mice. Obesity is one of the risk factors for NAFLD. One cause of obesity is characterized by adipocyte hypertrophy. Adipose tissue secretes many biologically active adipokines. The adipocytokines, such as adiponectin, are decreased in obese adolescents [42]. Hepatic stellate cells (HSCs) play a pivotal role in liver fibrosis [43]. Because adiponectin might suppress the proliferation and migration of HSCs and have biological significance in liver fibrosis, Kamada et al. tried to determine the role of adiponectin using adiponectin null mice and an adenovirusmediated adiponectin expression system. When mice were administered carbon tetrachloride to cause liver damage, the knockout mice showed liver fibrosis with increased expression of TGF- $\beta 1$ and connective tissue growth factor, while adenovirus-mediated adiponectin expression mice showed lower levels of liver fibrosis [44]. Two adiponectin receptors (adipoR1 and adipoR2) have been shown in various tissues, and adipoR2 is present predominantly in the liver [45]. Tomita et al. reported that adiponectin receptor AdipoR2 signaling in hepatocytes regulated the steatohepatitis progression by changing the peroxisome proliferator-activated receptor $\alpha$ (PPAR- $\alpha)$ activity and reactive oxygen species (ROS) accumulation [46].

\subsection{Adipocyte-Mediated Wnt Signaling: Secreted Frizzled-} Related Protein 5 (Sfrp5) knockout Mice. Adipose tissue releases adipokines, such as tumor necrosis factor $\alpha(\mathrm{TNF} \alpha)$, interleukine-6 (IL-6), and leptin. Many of them promote inflammation and disrupt glucose homeostasis [47]. Sfrp5, a protein linked to the Wnt signaling pathway in adipose tissue, is an anti-inflammatory adipokine expressed at higher levels in white adipose tissue [48]. Adenovirusmediated deliverly of Sfrp5 knockout mice fed a highfat and sucrose diet became obese and developed insulin resistance and hepatic steatosis. The development of insulin resistance is linked to macrophage-mediated inflammation. And activated macrophages, which accumulate in adipose tissue, are associated with the production of proinflammatory adipocytokines including TNF- $\alpha$ and IL-6. Moreover, the c-Jun N-terminal kinase signaling pathway, a downstream target of the noncanonical Wnt signaling, is activated [49].

\subsection{Adipocyte Apoptosis: BH3 Interacting Domain Death} Agonist (Bid) Null Mice. Adipocyte necrotic cell death with macrophage infiltration has been described in both obese humans and mice $[50,51]$. The protein Bid leads to apoptosis, regulating the signals to a cell death receptor known as Fas. However, Bid-deficient hepatocytes are resistant to Fasmediated apoptosis $[52,53]$. In Bid null mice, macrophage infiltration in adipose tissue was prevented, which protected against the development of systemic insulin resistance and hepatic steatosis associated with high-fat diet-induced obesity [54].

4.4. Fas Adipocyte-Specific Knockout Mice. Fas receptor, also known as CD95, a mediator of apoptosis, can regulate 
inflammatory pathways in several tissues. Fas expression was remarkably increased in the adipocytes of mouse models of obesity and insulin resistance. Mice with selective deletion of Fas were spared the deterioration of glucose homeostasis, insulin resistance, and hepatic steatosis that would otherwise be induced by a high-fat diet. Fas can also regulate inflammation, and the reduced adipocyte, IL6, CD11b, MCP1, and resistin mRNA levels were found in Fas knockout mice, while the levels of noninflammatory IL10 and arginase 1 were increased [55].

4.5. IL-6 and TNF Null Mice. The tumor-promoting cytokines IL- 6 and TNF caused by obesity are involved in the development of hepatic inflammation and steatosis. IL-6 and TNF null mice have decreased production of hepatic lipid and macrophage infiltration when fed a high-fat diet, resulting in the suppression of steatohepatitis and hepatocellular carcinoma (HCC) formation [56]. This mouse model supports the hypothesis that cytokines generated by adipose tissue can lead to insulin resistance, hepatic inflammation, and steatosis. Moreover, HCC is generated under inflammation and fatty liver in obesity.

4.6. Subacute "Inflammation" by Low-Level Activation of $N F-\kappa B$ in the Liver of Transgenic Mice, Designated LIKK Mice. NF- $\kappa \mathrm{B}$ signaling regulates a proinflammatory stage that controls the production of a host of inflammatory markers and cytokines, such as CRP, PAI-1, IL-6, TNF$\alpha$, and IL- $1 \beta$. I $\kappa \mathrm{B}$ kinase IKK- $\beta$ inhibits $\mathrm{NF}-\kappa \mathrm{B}$ activity. LIKK mice showed low level of activation of NF- $\kappa$ B in the livers by selectively expressing constitutively active IKK- $\beta$ in hepatocytes [57]. Moreover, the genetic overexpression of IKK- $\beta$ led to increased glucose levels and hepatic and systemic insulin resistance and increased hepatic production of proinflammatory cytokines. The mice had normal overall appearance in body weight, and their livers were histologically normal [57].

4.7. NF- $\kappa B$ Essential Modulator Gene (NEMO $\left.{ }^{L-K O}\right)$ Mice. Obesity results in a chronic inflammatory state, and adipose tissue releases proinflammatory cytokines such as TNF and IL-6. These cytokines lead to insulin resistance in skeletal muscle, liver, and adipose cells. Particularly, TNF-stimulated activation of the JNK and NF- $\kappa \mathrm{B}$ signaling pathways has been reported to inhibit insulin action [58]. TNF binding induces the activation of the IKK-1 and -2 kinases that are released from NEMO, thereby regulating the phosphorylation and degradation of $\mathrm{I}-\kappa \mathrm{Bs}$ liberated by the transcription factor NF- $\kappa \mathrm{B}[58,59]$. First, Luedde et al. reported that mice lacking NEMO in liver parenchymal cells spontaneously develop chronic hepatitis [60]. Then, glucose metabolism was analyzed in $\mathrm{NEMO}^{\mathrm{L}-\mathrm{KO}}$ mice exposed to a high-fat diet (HFD). These animals are protected from the development of obesity-associated insulin resistance. The authors have reported that hepatic NEMO deficiency with HFD develops in liver steatosis and liver tumorigenesis [61].

4.8. $\mathrm{NKK}^{-/-}$Mice. The c-Jun amino-terminal kinases (JNKs) that determine the biological outcome of TNF stimulation can interfere with the action of insulin [62]. Increased JNK activity can develop insulin resistance, and $\mathrm{JNK}^{-/-}$ mice have improved insulin sensitivity and enhanced insulin receptor signaling during obesity [63]. This suggests that JNK may have a role in developing steatosis. Indeed, Schattenberg et al. have shown that $\mathrm{JNK}^{-/-}$mice fed the MCD diet have significantly reduced steatohepatitis compared with wildtype mice [64].

\section{Conclusion}

This paper has described murine dietary and genetic models of NAFLD, NASH, and steatohepatitis. A number of animal models have been reported, and a variety of factors have been shown to induce fatty liver formation. These factors can induce systemic changes, steatosis, hepatic inflammation, and fibrosis. We cannot elucidate the pathogenesis of NASH using only one type of animal model. Steatosis should be considered as having a key role in the underlying disease process, but evidence has recently suggested that free fatty acids and their metabolites may be one of the major factors in the pathogenesis of NASH because their metabolites are highly toxic to the liver. The factors that potentiate the progression from simple steatosis to $\mathrm{NASH}$, some of which we have discussed, should be the focus of study.

\section{References}

[1] J. Ludwig, T. R. Viggiano, D. B. McGill, and B. J. Oh, "Nonalcoholic steatohepatitis: Mayo Clinic experiences with a hitherto unnamed disease," Mayo Clinic Proceedings, vol. 55, no. 7, pp. 434-438, 1980.

[2] M. Cave, I. Deaciuc, C. Mendez et al., "Nonalcoholic fatty liver disease: predisposing factors and the role of nutrition," Journal of Nutritional Biochemistry, vol. 18, no. 3, pp. 184-195, 2007.

[3] N. Assy, K. Kaita, D. Mymin, C. Levy, B. Rosser, and G. Minuk, "Fatty infiltration of liver in hyperlipidemic patients," Digestive Diseases and Sciences, vol. 45, no. 10, pp. 1929-1934, 2000.

[4] A. Sazci, G. Akpinar, C. Aygun, E. Ergul, O. Senturk, and S. Hulagu, "Association of apolipoprotein E polymorphisms in patients with non-alcoholic steatohepatitis," Digestive Diseases and Sciences, vol. 53, no. 12, pp. 3218-3224, 2008.

[5] E. M. Brunt, C. G. Janney, A. M. Di Bisceglie, B. A. Neuschwander-Tetri, and B. R. Bacon, "Nonalcoholic steatohepatitis: a proposal for grading and staging the histological lesions," American Journal of Gastroenterology, vol. 94, no. 9, pp. 2467-2474, 1999.

[6] D. E. Kleiner, E. M. Brunt, M. Van Natta et al., "Design and validation of a histological scoring system for nonalcoholic fatty liver disease," Hepatology, vol. 41, no. 6, pp. 1313-1321, 2005.

[7] L. Hebbard and J. George, "Animal models of nonalcoholic fatty liver disease," Nature Reviews Gastroenterology and Hepatology, vol. 8, no. 1, pp. 35-44, 2011.

[8] C. P. Day and O. F. W. James, "Steatohepatitis: a tale of two "Hits"?” Gastroenterology, vol. 114, no. 4, pp. 842-845, 1998.

[9] C. P. Day, "Pathogenesis of steatohepatitis," Best Practice \& Research in Clinical Gastroenterology, vol. 16, no. 5, pp. 663-678, 2002. 
[10] Q. M. Anstee and R. D. Goldin, "Mouse models in nonalcoholic fatty liver disease and steatohepatitis research," International Journal of Experimental Pathology, vol. 87, no. 1, pp. 1-16, 2006.

[11] L. A. Adams, J. F. Lymp, J. S. Sauver et al., “The natural history of nonalcoholic fatty liver disease: a population-based cohort study," Gastroenterology, vol. 129, no. 1, pp. 113-121, 2005.

[12] H. Cortez-Pinto, M. C. de Moura, and C. P. Day, "Non-alcoholic steatohepatitis: from cell biology to clinical practice," Journal of Hepatology, vol. 44, no. 1, pp. 197-208, 2006.

[13] H. Tilg and A. R. Moschen, "Evolution of inflammation in nonalcoholic fatty liver disease: the multiple parallel hits hypothesis," Hepatology, vol. 52, no. 5, pp. 1836-1846, 2010.

[14] E. F. Coyle, A. E. Jeukendrup, A. J. M. Wagenmakers, and W. H. M. Saris, "Fatty acid oxidation is directly regulated by carbohydrate metabolism during exercise," American Journal of Physiology, vol. 273, no. 2, pp. E268-E275, 1997.

[15] S. G. Sheth, F. D. Gordon, and S. Chopra, "Nonalcoholic steatohepatitis," Annals of Internal Medicine, vol. 126, no. 2, pp. 137-145, 1997.

[16] B. Cohen, D. Novick, and M. Rubinstein, "Modulation of insulin activities by leptin," Science, vol. 274, no. 5290, pp. 1185-1188, 1996.

[17] H. Satoh, M. T. A. Nguyen, P. D. G. Miles, T. Imamura, I. Usui, and J. M. Olefsky, "Adenovirus-mediated chronic "hyperresistinemia" leads to in vivo insulin resistance in normal rats," Journal of Clinical Investigation, vol. 114, no. 2, pp. 224-231, 2004.

[18] C. P. Day and S. Saksena, "Non-alcoholic steatohepatitis: definitions and pathogenesis," Journal of Gastroenterology and Hepatology, vol. 17, supplement 3, pp. S377-S384, 2002.

[19] F. Marra, A. Gastaldelli, G. Svegliati Baroni, G. Tell, and C. Tiribelli, "Molecular basis and mechanisms of progression of non-alcoholic steatohepatitis," Trends in Molecular Medicine, vol. 14, no. 2, pp. 72-81, 2008.

[20] R. Pais, A. Pascale, L. Fedchuck, F. Charlotte, T. Poynard, and V. Ratziu, "Progression from isolated steatosis to steatohepatitis and fibrosis in nonalcoholic fatty liver disease," Clinics and Research in Hepatology and Gastroenterology, vol. 35, no. 1, pp. 23-28, 2011.

[21] Y. Zhang, R. Proenca, M. Maffei, M. Barone, L. Leopold, and J. M. Friedman, "Positional cloning of the mouse obese gene and its human homologue," Nature, vol. 372, no. 6505, pp. 425-432, 1994.

[22] K. Yamaguchi, L. Yang, S. McCall et al., "Inhibiting triglyceride synthesis improves hepatic steatosis but exacerbates liver damage and fibrosis in obese mice with nonalcoholic steatohepatitis," Hepatology, vol. 45, no. 6, pp. 1366-1374, 2007.

[23] M. E. Rinella and R. M. Green, "The methionine-choline deficient dietary model of steatohepatitis does not exhibit insulin resistance," Journal of Hepatology, vol. 40, no. 1, pp. 47-51, 2004.

[24] M. E. Rinella, M. S. Elias, R. R. Smolak, T. Fu, J. Borensztajn, and R. M. Green, "Mechanisms of hepatic steatosis in mice fed a lipogenic methionine choline-deficient diet," Journal of Lipid Research, vol. 49, no. 5, pp. 1068-1076, 2008.

[25] I. A. Leclercq, G. C. Farrell, C. Sempoux, A. dela Peña, and Y. Horsmans, "Curcumin inhibits NF- $\kappa \mathrm{B}$ activation and reduces the severity of experimental steatohepatitis in mice," Journal of Hepatology, vol. 41, no. 6, pp. 926-934, 2004.
[26] A. dela Peña, I. Leclercq, J. Field, J. George, B. Jones, and G. Farrell, "NF- $\kappa$ B activation, rather than TNF, mediates hepatic inflammation in a murine dietary model of steatohepatitis," Gastroenterology, vol. 129, no. 5, pp. 1663-1674, 2005.

[27] J. Yu, E. Ip, A. dela Peña et al., "COX-2 induction in mice with experimental nutritional steatohepatitis: role as pro-inflammatory mediator," Hepatology, vol. 43, no. 4, pp. 826-836, 2006.

[28] C. Z. Larter, M. M. Yeh, J. Williams, K. S. Bell-Anderson, and G. C. Farrell, "MCD-induced steatohepatitis is associated with hepatic adiponectin resistance and adipogenic transformation of hepatocytes," Journal of Hepatology, vol. 49, no. 3, pp. 407-416, 2008.

[29] T. Nagasawa, Y. Inada, S. Nakano et al., "Effects of bezafibrate, PPAR pan-agonist, and GW501516, PPAR $\delta$ agonist, on development of steatohepatitis in mice fed a methionine- and choline-deficient diet," European Journal of Pharmacology, vol. 536, no. 1-2, pp. 182-191, 2006.

[30] D. Nakae, Y. Mizumoto, N. Andoh et al., "Comparative changes in the liver of female Fischer-344 rats after short- term feeding of a semipurified or a semisynthetic L-amino acid-defined choline-deficient diet," Toxicologic Pathology, vol. 23, no. 5, pp. 583-590, 1995.

[31] N. Matsuzawa, T. Takamura, S. Kurita et al., "Lipid-induced oxidative stress causes steatohepatitis in mice fed an atherogenic diet," Hepatology, vol. 46, no. 5, pp. 1392-1403, 2007.

[32] M. Marí, F. Caballero, A. Colell et al., "Mitochondrial free cholesterol loading sensitizes to TNF- and Fas-mediated steatohepatitis," Cell Metabolism, vol. 4, no. 3, pp. 185-198, 2006.

[33] F. R. Jornayvaz, V. T. Samuel, and G. I. Shulman, "The role of muscle insulin resistance in the pathogenesis of atherogenic dyslipidemia and nonalcoholic fatty liver disease associated with the metabolic syndrome," Annual Review of Nutrition, vol. 30, pp. 273-290, 2010.

[34] C. S. Lieber, M. A. Leo, K. M. Mak et al., "Model of nonalcoholic steatohepatitis," American Journal of Clinical Nutrition, vol. 79, no. 3, pp. 502-509, 2004.

[35] Y. Zou, J. Li, C. Lu et al., "High-fat emulsion-induced rat model of nonalcoholic steatohepatitis," Life Sciences, vol. 79, no. 11, pp. 1100-1107, 2006.

[36] X. Ouyang, P. Cirillo, Y. Sautin et al., "Fructose consumption as a risk factor for non-alcoholic fatty liver disease," Journal of Hepatology, vol. 48, no. 6, pp. 993-999, 2008.

[37] M. F. Abdelmalek, A. Suzuki, C. Guy et al., "Increased fructose consumption is associated with fibrosis severity in patients with nonalcoholic fatty liver disease," Hepatology, vol. 51, no. 6, pp. 1961-1971, 2010.

[38] A. Spruss, G. Kanuri, S. Wagnerberger, S. Haub, S. C. Bischoff, and I. Bergheim, "Toll-like receptor 4 is involved in the development of fructose-induced hepatic steatosis in mice," Hepatology, vol. 50, no. 4, pp. 1094-1104, 2009.

[39] E. Lopez-Garcia, M. B. Schulze, J. B. Meigs et al., "Consumption of trans fatty acids is related to plasma biomarkers of inflammation and endothelial dysfunction," Journal of Nutrition, vol. 135, no. 3, pp. 562-566, 2005.

[40] Q. Sun, J. Ma, H. Campos et al., "A prospective study of trans fatty acids in erythrocytes and risk of coronary heart disease," Circulation, vol. 115, no. 14, pp. 1858-1865, 2007.

[41] N. Obara, K. Fukushima, Y. Ueno et al., "Possible involvement and the mechanisms of excess trans-fatty acid consumption in severe NAFLD in mice," Journal of Hepatology, vol. 53, no. 2, pp. 326-334, 2010. 
[42] C. M. Halleux, M. Takahashi, M. L. Delporte et al., "Secretion of adiponectin and regulation of apM1 gene expression in human visceral adipose tissue," Biochemical and Biophysical Research Communications, vol. 288, no. 5, pp. 1102-1107, 2001.

[43] S. L. Friedman, "Seminars in medicine of the Beth Israel Hospital, Boston. The cellular basis of hepatic fibrosis. Mechanisms and treatment strategies," New England Journal of Medicine, vol. 328, no. 25, pp. 1828-1835, 1993.

[44] Y. Kamada, S. Tamura, S. Kiso et al., "Enhanced carbon tetrachloride-induced liver fibrosis in mice lacking adiponectin," Gastroenterology, vol. 125, no. 6, pp. 1796-1807, 2003.

[45] T. Kadowaki and T. Yamauchi, "Adiponectin and adiponectin receptors," Endocrine Reviews, vol. 26, no. 3, pp. 439-451, 2005.

[46] K. Tomita, Y. Oike, T. Teratani et al., "Hepatic AdipoR2 signaling plays a protective role against progression of nonalcoholic steatohepatitis in mice," Hepatology, vol. 48, no. 2, pp. 458-473, 2008.

[47] N. Ouchi, S. Kihara, T. Funahashi, Y. Matsuzawa, and K. Walsh, "Obesity, adiponectin and vascular inflammatory disease," Current Opinion in Lipidology, vol. 14, no. 6, pp. 561-566, 2003.

[48] Y. Kawano and R. Kypta, "Secreted antagonists of the Wnt signalling pathway," Journal of Cell Science, vol. 116, no. 13, pp. 2627-2634, 2003.

[49] N. Ouchi, A. Higuchi, K. Ohashi et al., "Sfrp5 is an antiinflammatory adipokine that modulates metabolic dysfunction in obesity," Science, vol. 329, no. 5990, pp. 454-457, 2010.

[50] K. J. Strissel, Z. Stancheva, H. Miyoshi et al., "Adipocyte death, adipose tissue remodeling, and obesity complications," Diabetes, vol. 56, no. 12, pp. 2910-2918, 2007.

[51] I. Murano, G. Barbatelli, V. Parisani et al., "Dead adipocytes, detected as crown-like structures, are prevalent in visceral fat depots of genetically obese mice," Journal of Lipid Research, vol. 49, no. 7, pp. 1562-1568, 2008.

[52] X. M. Yin, K. Wang, A. Gross et al., "Bid-deficient mice are resistant to Fas-induced hepatocellular apoptosis," Nature, vol. 400, no. 6747, pp. 886-891, 1999.

[53] S. Nagata, "Biddable death," Nature Cell Biology, vol. 1, no. 6, pp. E143-E145, 1999.

[54] N. Alkhouri, A. Gornicka, M. P. Berk et al., "Adipocyte apoptosis, a link between obesity, insulin resistance, and hepatic steatosis," Journal of Biological Chemistry, vol. 285, no. 5, pp. 3428-3438, 2010.

[55] S. Wueest, R. A. Rapold, D. M. Schumann et al., "Deletion of Fas in adipocytes relieves adipose tissue inflammation and hepatic manifestations of obesity in mice," Journal of Clinical Investigation, vol. 120, no. 1, pp. 191-202, 2010.

[56] E. J. Park, J. H. Lee, G. Y. Yu et al., "Dietary and genetic obesity promote liver inflammation and tumorigenesis by enhancing IL-6 and TNF expression," Cell, vol. 140, no. 2, pp. 197-208, 2010.

[57] D. Cai, M. Yuan, D. F. Frantz et al., "Local and systemic insulin resistance resulting from hepatic activation of IKK- $\beta$ and NF$\kappa$ B," Nature Medicine, vol. 11, no. 2, pp. 183-190, 2005.

[58] P. Plomgaard, K. Bouzakri, R. Krogh-Madsen, B. Mittendorfer, J. R. Zierath, and B. K. Pedersen, "Tumor necrosis factor- $\alpha$ induces skeletal muscle insulin resistance in healthy human subjects via inhibition of Akt substrate 160 phosphorylation," Diabetes, vol. 54, no. 10, pp. 2939-2945, 2005.

[59] G. S. Hotamisligil, P. Peraldi, A. Budavari, R. Ellis, M. F. White, and B. M. Spiegelman, "IRS-1-mediated inhibition of insulin receptor tyrosine kinase activity in TNF- $\alpha$ - and obesity-induced insulin resistance," Science, vol. 271, no. 5249, pp. 665-668, 1996.

[60] T. Luedde, N. Beraza, V. Kotsikoris et al., "Deletion of $\mathrm{NEMO/IKK} \gamma$ in liver parenchymal cells causes steatohepatitis and hepatocellular carcinoma," Cancer Cell, vol. 11, no. 2, pp. 119-132, 2007.

[61] F. T. Wunderlich, T. Luedde, S. Singer et al., "Hepatic NF$\kappa \mathrm{B}$ essential modulator deficiency prevents obesity-induced insulin resistance but synergizes with high-fat feeding in tumorigenesis," Proceedings of the National Academy of Sciences of the United States of America, vol. 105, no. 4, pp. 1297-1302, 2008.

[62] J. K. Sethi and G. S. Hotamisligil, “The role of TNF $\alpha$ in adipocyte metabolism," Seminars in Cell and Developmental Biology, vol. 10, no. 1, pp. 19-29, 1999.

[63] J. Hirosumi, G. Tuncman, L. Chang et al., "A central, role for JNK in obesity and insulin resistance," Nature, vol. 420, no. 6913, pp. 333-336, 2002.

[64] J. M. Schattenberg, Y. Wang, R. Singh, R. M. Rigoli, and M. J. Czaja, "Hepatocyte CYP2E1 overexpression and steatohepatitis lead to impaired hepatic insulin signaling," Journal of Biological Chemistry, vol. 280, no. 11, pp. 9887-9894, 2005. 


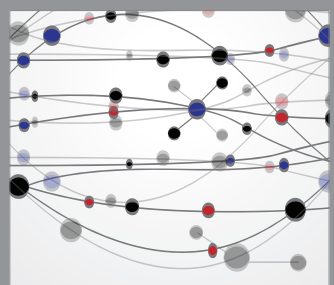

The Scientific World Journal
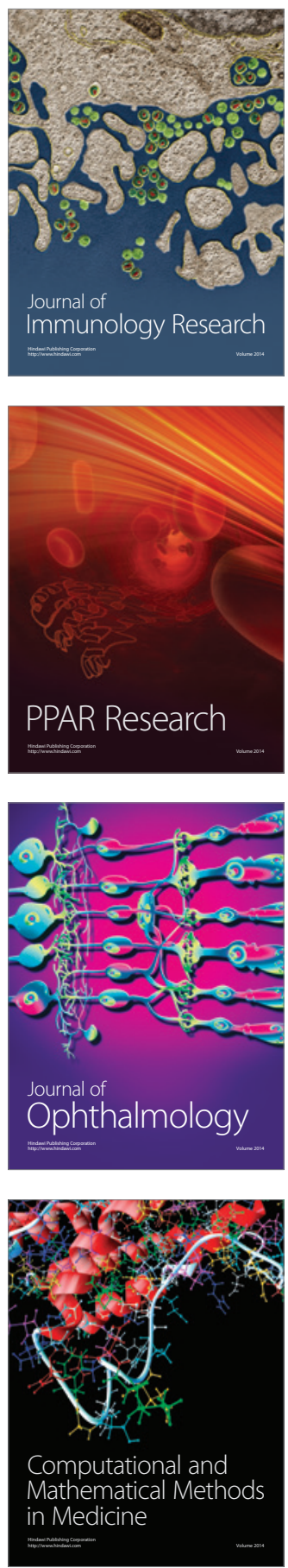

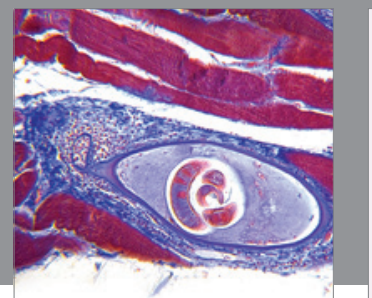

Gastroenterology

Research and Practice
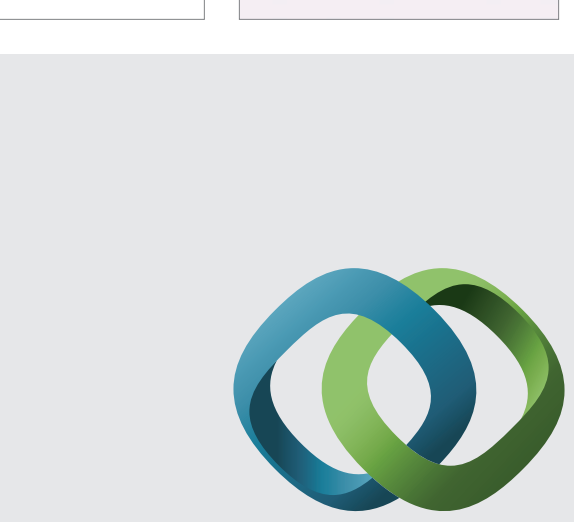

\section{Hindawi}

Submit your manuscripts at

http://www.hindawi.com
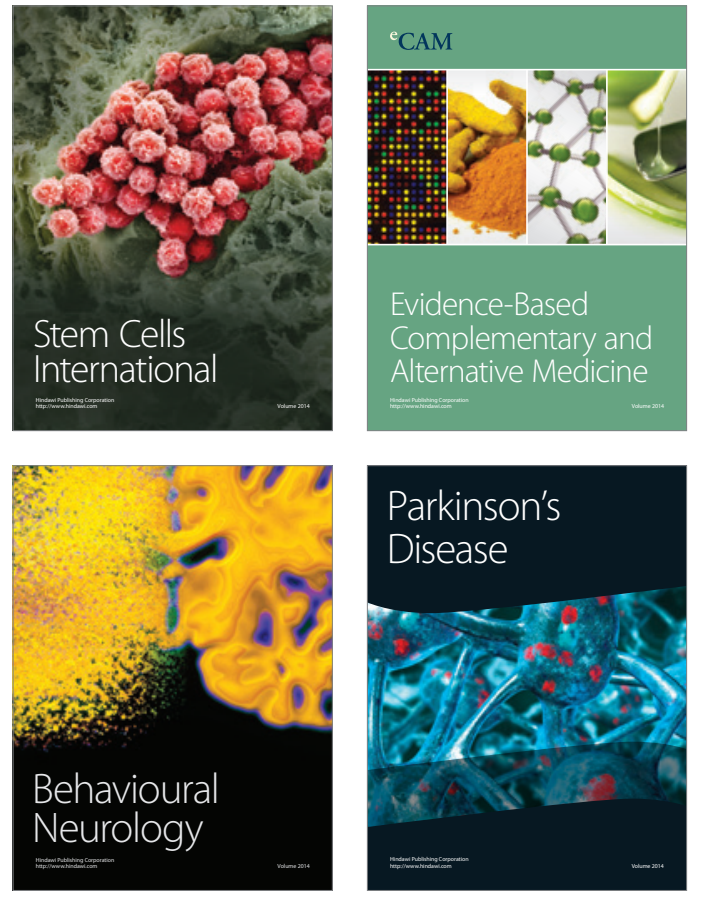
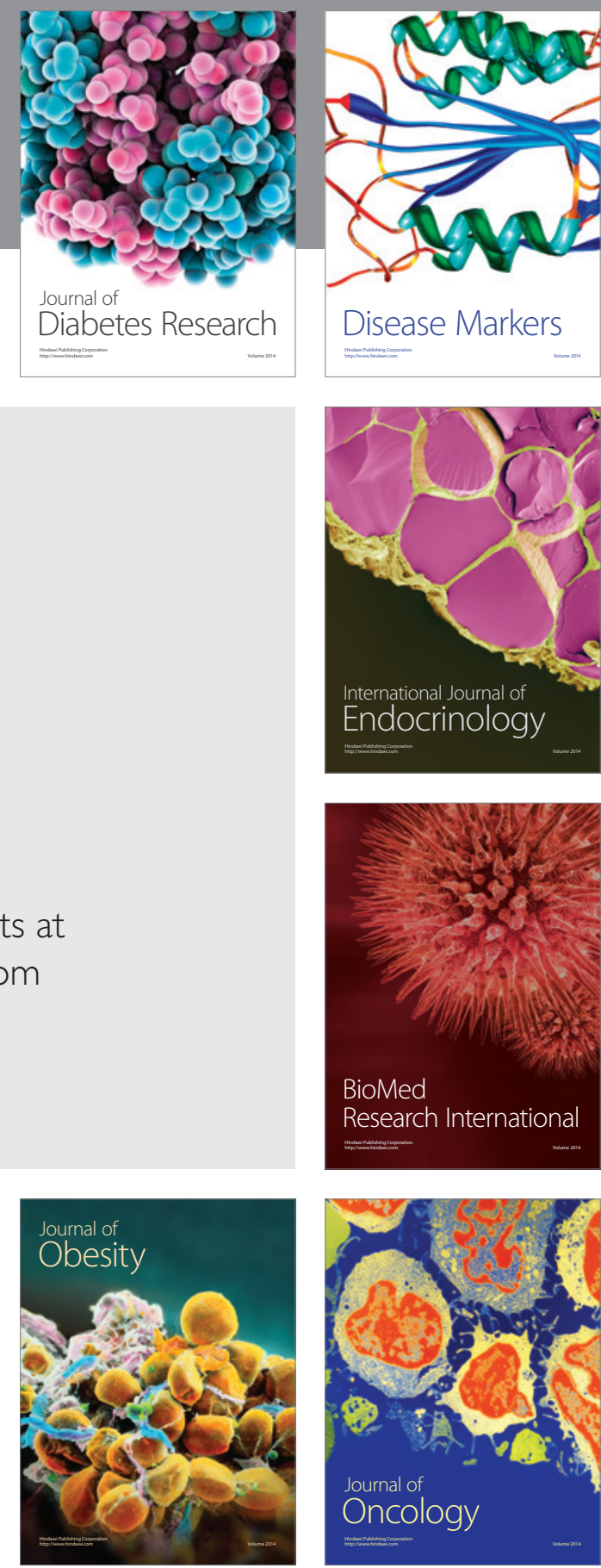

Disease Markers
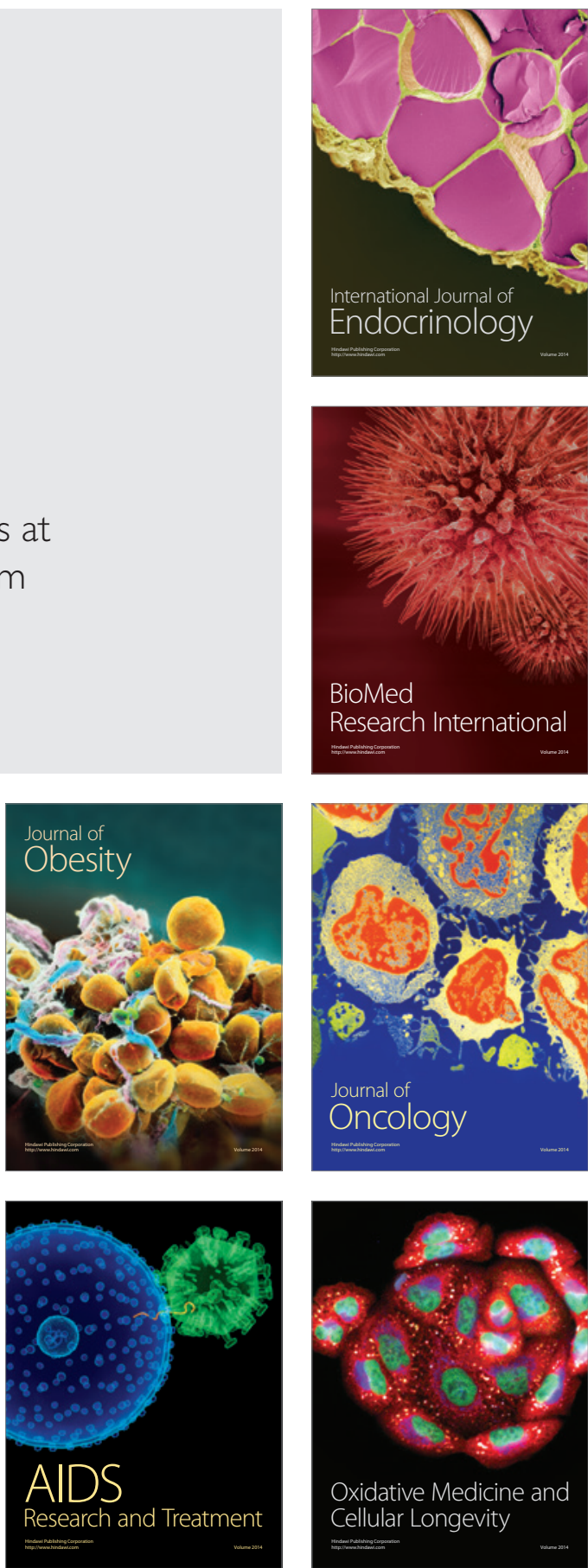